\title{
Software Process Improvement
}

\section{in Multimodel Environments}

\author{
André Ferreira \\ Department of Information Systems \\ University of Minho \\ Guimarães, Portugal \\ andre.ferreira@dsi.uminho.pt
}

\author{
Ricardo Machado \\ Department of Information Systems \\ University of Minho \\ Guimarães, Portugal \\ rmac@dsi.uminho.pt
}

\begin{abstract}
Software is increasing in size and complexity. As a direct implication, organizations find it more difficult to achieve the desired product quality. Process improvement models are used to improve product quality. In recent years the combination of different improvement models is increasing. The main challenge in these environments is the interoperability of chosen models. An incorrect integration approach diminishes efficiency and effectiveness on applying these models. The general objective of this thesis is to identify principles and process characteristics for designing a system of processes at the architectural level. As a result of this research work, an improved understanding of improvement models interoperability is expected by identifying the technical and structural relationships between processes that will help architecting a system of processes.
\end{abstract}

Software Engineering Management; Software Engineering Process

\section{INTRODUCTION}

Services that rely on software are now pervasive in today's society. These range from simple electronic shopping services to software responsible for flying airplanes and manage nuclear power plants safety. Software is being used in a wide number of knowledge areas and applications are becoming more complex as the functionality required to provide services is evolving. An evidence is the increasing number of lines of code per application [1]. As software system are becoming even larger and complex, defects will be part of the increasing lines of code that bring these systems to work. Software quality is a concern as systems are growing in complexity. Many examples of failures exist that resulted from software problems caused by defects that have been overlooked, these are associated to ineffective development practices, and poor management decisions [2].

A software process can be defined as the logical organization of people, materials, energy equipment, and procedures into work activities designed to produce a specified end result [3]. The result expected involves satisfying cost estimates, schedules and required quality attributes with some consistency. People and technology are integral parts in this process and perform a fundamental role in the resulting product quality. To sustain an acceptable level of consistency the software process must be effective most of the times, meaning there is an end product resulting from the process. This is not always the case with an $a d-h o c$ approach, characterized by being highly dependable on external factors like competence and extraordinary efforts by people in the organization. When this external conjecture fails the product frequently is not delivered. To be effective a process must be planned and executed with accordance to a policy, comprehending a first level of achievable control.

A common organizational strategy to achieve some level of control in product quality is to use appropriate processes and methods in product development. Processes and methods are viewed as control levers in product quality, to improve quality the process must be constantly improved. Organizations begin their process improvement when they find product issues in the field and start investigating how to prevent similar issues to occur in the future.

Software Process Improvement (SPI) approaches popular in software industry are the CMMI (Capability Maturity Model Integrated) [4] and ISO Standard 9001 [5]. The former also addresses other domains, e.g., systems engineering, integrated product and process development and supplier outsourcing. The later is a general purpose standard to be applicable across industry. Both are being used to address the challenge of improving the software process and stories of success of applying models to obtain these benefits are increasing [6-8].

Nevertheless, SPI initiatives are not easy to implement, they carry significant risk of failure [9]. The complexity of undertaking SPI requires commitment, proper skills and resources, these all need to be assured to carry SPI with success. Specifically, when planning for multimodel environments, organizations find that models often overlap in the requirements they define to implement the best practices they advocate. Selection of the most adequate models becomes an issue. Additionally, their integration is not always clear as the granularity of their descriptions and scope of applicability vary considerably. These issues when improperly addressed can lead to inefficiency in model integration, eroding the benefits of these SPI efforts [10].

In this context methods and techniques that help in the selection, design and implementation of multimodel solutions are required to help organizations reduce risks when approaching SPI in multimodel environments.

\section{STATE OF THE ART}

SPI efforts are systematically justified by the endless quest of achieving competitiveness advantage in customer 
satisfaction, business profitability, market share, product and service quality, cost reduction, cycle time reduction, etc.

A long list of models, standards, best practices, regulatory policies and other types of practices aim to provide the necessary guidelines to help organizations guide these efforts. The term improvement technologies was coined by Siviy in [10] and will be used interchangeably as shorthand for this list of artefacts that help guide improvement efforts. Improvement technologies provide unique features to address specific problems these vary in scope and domain of applicability. A simple taxonomy classifies technologies in two groups, the ones that are oriented to answer 'what' needs to be done and the ones that show 'how' it could be done. When organizations adopt a multimodel approach they aim to leverage the best practices made available by different models to better address improvement challenges.

Common approaches combine CMMI \& Six Sigma [1114] and CMMI \& ISO [15-17] with the purpose of developing a single integrated solution.

The value proposition of using multiple technologies is the incorporation of their best practices in a single solution, that otherwise would not be possible to obtain by a single technological approach. Two general approaches are being considered when combining different technologies, these fall in what/how and what/what combinations. Examples of technologies that show what needs to be done are CMMI and ISO 9001. Six Sigma, Team Software Process [18], Personal Software Process [19] and Project Management Body of Knowledge provide answers on how it could be done.

Nevertheless, a heterogeneous environment with multiple overlapping technologies used to develop improvement initiatives raises several problems. One of the issues with multimodel environments is the competition between technologies when several initiatives are concurrently implemented at difference hierarchical levels [10]. This leads to competition over resources by the different approaches to satisfy their specific needs. The overlapping efforts are costly and the benefices of each technological approach are undermined by the conflicting approaches. Additionally, technologies are stove-piped along the years of improvement efforts motivated by different improvement needs. This approach is most of the times affected by lack of coordination in the integration of the existing and new approaches leading to an unplanned multi-model environment. This results in lack of effectiveness of the multiple technological approaches, where redundancies and unrealized synergies between competing technologies become apparent.

The current state of the art in SPI in multimodel environments is best described using an underlying framework that defines the steps required to guide SPI efforts in multimodel environments. This framework defined in [10] provides a comprehensive description of the steps that guide the development and implementations of improvement initiatives. In order to leverage the use of multiple technologies and manage their complex integration, a harmonized approach based in a reasoning framework for multimodel harmonization is proposed. The reasoning framework guides the development of an appropriate solution to meet organizational objectives by understanding and leveraging the technological properties of interest, as well as composing these properties in process architecture to create a harmonized solution.

The reasoning framework is composed by a set of basic steps, questions to be addressed and principles to use in the process of alignment of the layers and levels of an integrated business. In summary the reasoning framework defines four steps. The first step focus in mission translation and is based in the argument that business drivers should govern the selection of each improvement technology. The second step deals with technology selection, here strategic categorization of technologies is performed and used to help choose the best suited technologies that support business drivers. The third step deals with solution implementation where the selected technologies are composed and process architecting is performed to develop the actual organizational standard processes. The last step involves implementing the multimodel process improvement solution and measuring results.

The last three steps in the reasoning framework define the scope of the newest approaches being developed by organizational process improvement groups. They represent the current state of the art on SPI efforts in multimodel environments. Within the set of practices defined in the reasoning framework the ones that involve alignment of organizational and improvement objectives, solution deployment and measurement and analysis are not unique in multimodel situations. On the other hand technology selection and strategies, and solution implementation are tightly connected to multimodel SPI, and these are considered as new research topics [14].

On the topic of technology selection and strategy, the selection process is the focus of research. The decision of simultaneous adopting multiple technologies is not always easy. Technologies may address the same needs and those that address different needs may significantly overlap. Also, the way these are implemented influences the decision process. To help in multimodel decision making a few approaches may be considered, namely: affinity groupings of models and technologies; selection and implementation patterns of models, standards and other technologies; and rigorous decision methods.

Halvosen et al. [20] address the problem of comparison and selection of SPI frameworks. A taxonomy is defined to objectively compare SPI frameworks and to facilitate information gathering. The taxonomy aims to be used as a tool by organizations in the decision process about which frameworks to use, helping to overcome the difficulty of comparing SPI frameworks due to their comprehensive nature and diverse scope. Also, terms about software quality and software process are not consistent across frameworks, this requires individual interpretation regarding these concepts. The taxonomy developed uses the comparative characteristics method and comprises 25 characteristics grouped in five categories. It is influenced by previous work on the topic and tries to capture general aspects regarded as important. 
A different approach in the topic of technology selection is presented by Siviy et al. [14] by using an affinity matrix (see Figure 1) that groups models by their strategic value and application focus.

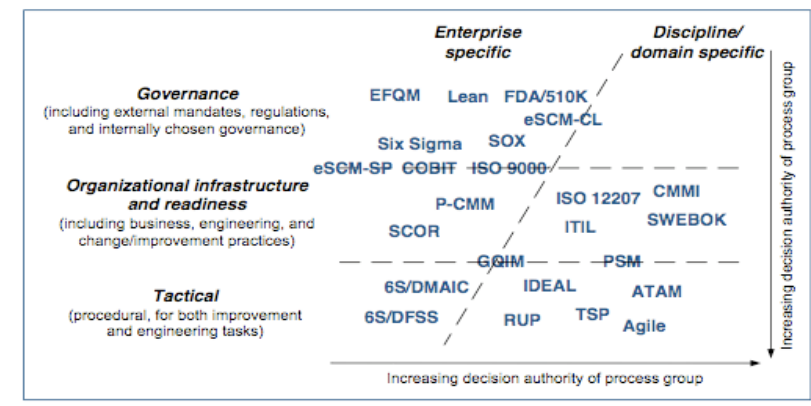

Figure 1 - Strategic Classification Taxonomy [14].

Additionally, indicates typical decision authority on models selection and can be used for pattern selection analysis. The matrix may be used in several ways; it can be used to link mission translation to its strategy and when combined with descriptions of model relationships can be used to develop the multimodel strategy.

Following to the topic of solution implementation Srivastava and Murthy [12], working for Tata Consulting Services developed an integrated approach using CMMI and Six Sigma. The initial objective was to develop a Quality Framework to address specific strategic challenges of the organization. The choice was to integrate CMMI and Six Sigma, where CMMI provided the 'what' to do and the Six sigma provided the 'how' to do it. The integration examples provided show a mapping process between CMMI process areas and applicable Six Sigma tools and, the integration of DMDAV (Define Measure Analyze Design and Verify) framework with the development phases defined in the organizational quality framework.

The mapping process between Six Sigma and CMMI process areas involved the selection and use of Six Sigma tools to address the specific goals of each CMMI PA (Process Area), e.g., the Quantitative Project Management PA defines the specific goal of statistically manage process performance, Six Sigma provides control charts to help identify special causes of variation. The relevance of the synergy is that by identifying the relevant causes of variations in project management sub-processes is possible to manage the process performance. The mapping process incorporates the relevant Six Sigma tools to be used in the process of elaborating the deliverables for each development phase, e.g., the Requirements Analysis uses the Affinity Diagram, the Quality Function Deployment and Critical To Quality Drill Down Tree to help translate the voice of customer to elaborate the System Requirements Specification. The Detailed Design, Build \& Test phases uses a wide set o Six Sigma tools, namely: Control Impact Matrix, Cost Benefit Analysis, Design of Experiments Simulation and Modelling, Failure Modes and Effects
Analysis and Validation Plans to help elaboration of low level design, unit test plans, unit test specifications and code.

The authors emphasize the rigor that Six Sigma provides by the use of DMADV framework and Six Sigma tools. These had a direct impact in defect reduction and process improvements that increased considerably cost savings.

Another work in the topic of solution implementation is presented by Mutafelija et. al [21]. The authors address the opportunity of combining the CMMI and SWEBOK (Software Engineering Body of Knowledge) to develop an organization set of standard processes and refer to the necessity of defining process architecture as a building block for the definition of a process definition.

The approach to integrate CMMI with SWEBOK had the objective to leverage the two approaches, in which CMMI provides 'what' to do and the SWEBOK provides 'how' to do it. CMMI outlines the process steps and the SWEBOK provides process details, additional references and further reading. The integration effort, where CMMI requires the definition of process elements and SWEBOK provides the implementation details, serves as motivation to elicit a process architecture or framework to leverage the multimodal approach for processes definition.

The authors address the issues of which should be the building blocks to be used for process definition. They provide some insights on characteristics that can be considered when defining process elements, where process elements are defined as the fundamental unit of a process and each process element covers a closely related set of activities [4]. Within these basic building blocks are task descriptions (functional), when and how tasks are performed (behavioural), who performs the tasks (organizational) and the guiding principles and strategies (meta architecture). Complementing the process characteristics are the process elements components, namely: entry and exit criteria, inputs and outputs, activities, roles and responsibilities, stakeholders, measurements, controls (verification, configuration management), related processes and tools, standards and training.

A different approach is presented by Sivie et.al [14] by introducing a synergetic relationship of the CMMI and Six Sigma at a different level. It uses CMMI process areas and Six Sigma frameworks, namely: Define Measure Analyze Improve and Control (DMAIC) and Define, Measure, Analyze, Design, Verify (DMADV) and Lean [22] to define design connectivity strategies to provide a more general solution adaptable to an organizational defined process. Trough, it does not provide a comprehensive description of the approach.

An example in design connectivity is presented between process areas and framework phases. The process areas Measurement and Analysis, Quantitative Project Management, Causal Analysis and Resolution, and Organizational Process Performance are mapped with to DMAIC roadmap steps. An example is given of this connectivity by using a measurement and analysis abstract process, defined in a set of generic steps. The steps in the measurement and analysis abstract process responsible for gather and analyze data are associated with the Measure step 
in DMAIC and are overlaid with the Measurement and Analysis process area of CMMI. In a similar fashion the generic step of conduct causal analysis in the abstract process is associated with the Analyze step of DMAIC and overlaid with Causal Analysis and Resolution process areas of CMMI. In the view presented by the authors on design connectivity's between Six Sigma and CMMI, DMAIC shares common and complementary characteristics with high maturity process areas, DFSS (Design for Six Sigma) with Engineering process areas and Lean with Project and Process Management process areas.

In a multimodel environment, improvement efforts require careful planning and integration as the use of competitive technologies may lead to misuse of resources. How to effective plan and integrate different technologies is one of the challenges being addressed by organizations. The harmonization framework developed by Sivie et al. and the Process Improvement in Multimodel Environments (PrIME) [23] project are representative of the interest and efforts in SPI on multimodel environments. The steps defined in the framework conceptualize the activities required to develop these SPI efforts. Technology selection and strategies and design of improvement solutions steps in this framework are tightly related to activities required in this multimodel context. These activities are challenges to organizations as state of practice is not yet achieved. Currently, an understanding of the architectural characteristics is insufficient to inform effective process composition with existing models and standards.

The concept of process architecting and the role of process architect seems to be gaining relevance as the system of processes is becoming more complex [24]. The functions, properties and building blocks of these systems of processes require precise definition. Further research work is required.

\section{RESEARCH OBJECTIVES AND APPROACH}

Process improvement groups deal with increasing complexity as interactions between emergent technologies are still not very well documented. Additionally, increasing demand for compliance with models and regulations demand robust and flexible process systems. The process composition activity is by these reasons complex, specifically if there is no access to experienced resources.

The organizational environment where research is to be undertaken develops critical mission software. To have access to these markets, contractors are often required to comply with multiple standards and certifications. In this context a multimodel environment is a necessity as it is a strategical approach to business. In order to address these demands Critical Software S.A. (CSW) has a Quality Management System (QMS) that complies with practices and requirements from multiple models such as CMMI, ISO9001, ISO 15504 [25] and ISO 12207 [26]. The former was designed to enable projects and other organizational units to achieve their objectives by providing the adequate processes and activities. CSW is now developing efforts to achieve CMMI level 5 and is introducing Six Sigma as a strategic approach to establish the high level processes of CMMI.

Several questions were raised about the ability of the QMS to serve its purpose as the organization is evolving and growing. Internally, the decision to introduce Six Sigma as a tactical governance model remains a challenge. The QMS complexity and size are considerable and a clear operational strategy for the introduction of this governance model is being defined.

Additionally, several issues are being raised regarding the attributes that a system of processes must exhibit to cope with change and growth. Complexity, agility, robustness and the ability to control costs were identified as attributes that deserve special attention. Guided by the necessity to provide answers to these issues, the following research goals are proposed:

\section{A. Identification and characterization of architectural elements}

In the process of developing the QMS, technologies were tailored/instantiated to form the set of organizational processes. Processes were designed and develop with certain characteristics and process components. Following the work of Mutafelija and Stromberg [21] on process architecture, research will be conducted to identify and characterize the necessary architectural elements that will enable an effective process composition and architecture. Possible features to consider are: functional properties, including classes flows and attributes; inputs and outputs, including flow and relationships; information flow; overall interrelationships, dependencies and constraints; and roles and responsibilities including users and actors.

The objective is to identify and characterize a set of basic architectural building blocks for process architecting in a context of composed combinations of technologies. The work on classes of comparison methods for SPI frameworks described by Halvorsen et al. [20] will be used as a starting research approach. Secondly, an analysis of the models that underpin the QMS structure will be conducted to identify the structural elements that supported the exercise of designing the overall solution. Thirdly, compare the identified elements with frameworks of software process modelling concepts like Object Management Group SPEM (Software Process Engineering Metamodel) and the OPEN Process Framework (OPF) and suggest possible extensions that support the rationale of the identified architectural elements.

The aim of this goal is to answer the question: Which are the features and properties to be considered when developing process architecture?

\section{B. Elaborate on technology composition}

Informed by the artefacts developed in the previous step, that provide a detailed description of how technologies can be composed based on architectural characteristics, it is now 
possible to investigate on possible technological compositions. A set of exercises will be conducted using the results obtained in the previous step. These exercises will consider the following scenarios: the first will use technologies that exhibit a high degree of semantic overlapping. A second scenario will include technologies that exhibit some degree of compromise with regard to recommended practices. A last exercise will consider technologies where attributes of flexibility and adaptability are required characteristics for a final solution, allowing addition and removal of blocks that compose the process system.

Six Sigma has demonstrated to be useful in several environments similar to CSW environment and is being integrated into the QMS as a strategic approach to achieve CMMI level 5 certification. Efforts will be developed to research on design connections between Six Sigma and high maturity process areas (specifically level 4 and 5 process areas of CMMI). The aim of this goal is to: suggest design connections between Six Sigma and other technologies that compose the QMS.

\section{Elaborate on process architecting}

The activity of process architecting underpins the organizational set of standard processes. It instantiates the reasoning and steps executed by the process architect to develop the actual solution. The efforts on this research goal will assume the activity of process architecting can be informed and guided by the composition of models and standards, and additionally aided by the structuring blocks for a system of processes. The research efforts will consider approaches and techniques that are being used inside and outside of the engineering disciples. Primarily and related to software and related engineering technologies the Quality Attribute Workshop and the Attribute-Driven Design [27] will be studied. From Six Sigma, DFSS, and Design for Lean Six Sigma. Outside the engineering disciplines, architectures and models for business process management will be analyzed.

These approaches will be studied and experimented to assess their applicability in the exercise of process architecting. A set of guidelines or method to guide the process architecting activity is expected to be developed. The question addressed by this goal is: how can be process architecture derived from technology classification and composability?

\section{Validate the research results in the previous steps}

The method will be developed and experimented iteratively in the organizational environment by being applied in the reengineering and evolution of the organizational QMS. This experimentation will serve the purpose of evaluate the usefulness of the research work.
Action Research [28, 29] will be used as research method to validate the research results. This method falls in the group of qualitative research methods and is considered a valuable approach by enabling a synergetic association between practice and theory. The method consists in a iterative process that involves researchers and practitioners in a cycle of activities, that includes problem diagnosing, action intervention and reflective learning [30]. For external visibility and validity three conferences were chosen as possible targets for publishing the research findings, namely the European Software Process Improvement Initiative EuroSPI [31], the EUROMICRO Conference on Software Engineering and Advanced Applications (SEAA) [32], in particular the track Software Process and Product Improvement and the International Symposium on Empirical Software Engineering and Measurement [33].

\section{CURRENT WORK AND PRELIMINARY RESULTS}

Work developed to date focused on activities performed as a member of the quality department at CSW. Efforts have been put in the execution of tasks set to define the new CMMI level 4 and level 5 processes areas. A high maturity process framework is being designed to incorporate the CMMI Level 4 and Level 5 process areas in the current QMS. Currently the challenges on multimodel integration are being felt as the QMS processes follow basically the structure provided by ISO 15504 and ISO 12207 process models. CMMI high maturity practices need to be built in to this structure. An integration strategy is being defined. Additionally, an analysis of the structure of the QMS is being carried out to identify and characterize the interprocess relations, processes building blocks and features, namely: functional properties, relationships, information flow dependencies and constraints. A literature review on the subject of SPI in multimodel environments has been performed.

\section{WORK PLAN AND IMPLICATIONS}

Based on the research objectives presented the work will consider three phases. The first phase has begun in the first year, as a result it will deliver a proposal of set of basic elements for process architecting. These will provide a work basis for approaching the second research objective on technology composition, as a practical approach for using the research results related to the first goal. The second phase will begin in the second year and will deliver an approach on design connections between Six Sigma and the technologies that underpin the organizational QMS. The final phase and final year will focus on elaboration on process architecting with the main goal of delivering a method and/or guidelines for organizations to address the complexity of process architecting a system of processes, with multiple model requirements. At the end of each phase, the research work shall be submitted for acceptance on main international conferences on the areas of research and will form the chapters of the $\mathrm{PhD}$ thesis. 
As original contribution to the body of knowledge for Software Engineering we expect to deliver a methodological approach for designing systems of processes. This will enable organizations to cope with the complexity of composing models and standards in multimodel environments. The guidance will focus on designing systems of processes at the architectural level, rather than the procedural level.

\section{CONCLUSION}

Organizations are considering multiple models to guide their SPI efforts. They aim to leverage the benefits of each model to develop a single harmonized integrated solution. These multimodel environments bring new challenges that organizations need to tackle. Risks associated are the misuse of resources and inefficient model integration. Inefficient integration undermines the benefits of each model and increases operational costs. Improvement technologies selection and strategy and solution implementation gain emphasis in this multimodel context. The system of processes is becoming more complex as new technologies are being created by the international bodies and organizations are required or chose to integrate them to improve their daily practices.

The current state of the art in the topic of technology selection is characterized by selection techniques, e.g., affinity matrix and taxonomies for comparing SPI frameworks. In the topic of solution implementation, model composition and process architecting activities are being carried with the objective of developing a single integrated solution. Design connections and technology mappings characterize the current state of the art in this topic. State of the art practices in multimodel environments emerge on organizations that are developing internal solutions. Recent work on these topics is made available at conferences that target practitioners and professionals. This thesis aims to combine the practice of developing solutions to be used in the organizational context and the efforts of applying a qualitative research method to provide original contribution to the body of knowledge on SPI. SPI is considered an experimental and practical field where research requires experimentation, data retrieval and analysis.

\section{REFERENCES}

[1] P. Miller, "An SEI Process Improvement Path to Software Quality," Quality of Information and Communications Technology, 2007. QUATIC 2007. 6th International Conference on the, pp. 12 - 20, Aug 312007.

[2] C. Jones, "Our worst current development practices," Software, IEEE, vol. 13, pp. 102-104, 1996

[3] W. A. Florac and A. D. Carleton, "Measuring the Software Process," Addison-Wesley, 1999.

[4] M. B. Chissis and M. Konrad, "CMMI for Development, Version 1.2," Addison-Wesley, 2006.

[5] "ISO/IEC 9001 Quality management systems - Requirements," ISO/IEC, 2000.

[6] H. Conradi and A. Fuggetta, "Improving Software Process Improvement," Software, IEEE, vol. 19, pp. 92- 99, 2002.

[7] F. McGarry and B. Decker, "Attaining Level 5 in CMM process maturity," Software, IEEE, vol. 19, pp. 87- 96, 2002.
[8] L. Mathiassen, O. Ngwenyama, and I. Aaen, "Managing change in software process improvement," Software, IEEE, vol. 22, pp. 84- 91, 2005.

[9] I. Aaen, "Software process improvement: Blueprints versus recipes," Software, IEEE, vol. 20, pp. 86 - 93, Sep 12003.

[10] J. Siviy, P. Kirwan, J. Morley, and L. Marino, "Maximizing your Process Improvement ROI through Harmonization," SEI http://www.sei.cmu.edu/prime/index.html - white paper, 2008.

[11] T. Pyzdek, "The Six Sigma Handbook," McGraw-Hill, 2003.

[12] N. Srivastava and S. Murthy, "Harvesting CMMI Benefits - The Six Sigma Sickle," SEPG Conference, 2006.

[13] R. Hefner and M. Sturgeon, "Optimize Your Solution: Integrating Six Sigma and CMM/CMMI-Based Process Improvement," Software Technology Conference, 2002.

[14] J. Siviy, M. Penn, and R. W. Stoddard, "CMMI and Six Sigma Partners in Process Improvement," Addison-Wesley, 2008.

[15] I. Rozman, R. Vajde Horvat, and J. Gyorkos, "United view on ISO 9001 model and SEI CMM," Engineering Management Conference, 1994. 'Management in Transition: Engineering a Changing World', Proceedings of the 1994 IEEE International, pp. 56 - 63, Sep 30 1994.

[16] B. Mutafelija and H. Stronberg, "Systematic Process Improvement Using ISO 9001:2000 and CMMI," Artech House, 2003.

[17] B. Mutafelija and H. Stronberg, "Mappings of ISO 9001:2000 and CMMI Version 1.1," SEI http://www.sei.cmu.edu/cmmi/adoption/iso-mapping.html - Access in November 2008, 2003.

[18] W. S. Humphrey, TSP(SM): Coaching Development Teams: Addison Wesley, 2006.

[19] W. S. Humphrey, PSP(SM): A Self-Improvement Process for Software Engineers: Addison Wesley, 2005.

[20] C. P. Halvorsen and R. Conradi, "A Taxonomy to Compare SPI Frameworks,"

http://www.idi.ntnu.no/grupper/su/publ/pdf/ewspt2001spiframework-final.pdf-Access in November 2008, Jan 12001.

[21] B. Mutafelija, "Architecting Standard Processes with SWEBOK and CMMI," SEPG Conference, 2006.

[22] M. Poppendieck and T. Poppendieck, "Lean Software Development: An Agile Toolkit," Addison-Wesley, 2003.

[23] "Process Improvement in Multimodel Environments (PrIME)," http://www.sei.cmu.edu/prime/primedesc.html - Acessed in December 2008.

[24] J. Kasser, "Introducing the Role of Process Architecting," The 15th International Symposium of the International Council on Systems Engineering (INCOSE), 2005.

[25] "ISO/IEC 15504 Software Process Improvement and Capability Determination Model (SPICE)," ISO/IEC, 2004.

[26] "ISO/IEC 12207 - Systems and software engineering - Software life cycle processes," ISO/IEC and IEEE, 2008.

[27] Nord, Wood, and Clements, "Integrating the Quality Attribute Workshop (QAW) and the Attribute-Driven Design (ADD) Method," CMU/SEI-2004-TN-017, 2004

[28] N. Kock, R. J. McQueen, and J. L. Scott, "Can action research be made more rigorous in a positivist sense? The contribution of an iterative approach," Journal of Systems and Information Technology, Jan 11997.

[29] H. B. Christensen, K. M. Hansen, and K. R. Schougaard, "Ready! Set! Go! An Action Research Agenda for Software Architecture Research," Software Architecture, 2008. WICSA 2008. Seventh Working IEEE/IFIP Conference on, pp. 257 - 260, Jan 312008.

[30] D. E. Avison, F. Lau, M. D. Myers, and P. A. Nielsen, "Action research," Communications of the ACM, vol. 42, Jan 11999.

[31] "European Software Process Improvement Initiative," http://www.isqi.org/en/conferences/eurospi/ - Access in December 2008.

[32] "EUROMICRO," http://www.euromicro.org/ - Access in November 2008.

[33] "International Symposium on Empirical Software Engineering and Measurement," http://www.esem-conferences.org/, 2008. 